This document is confidential and is proprietary to the American Chemical Society and its authors. Do not copy or disclose without written permission. If you have received this item in error, notify the sender and delete all copies.

\title{
Molecular Dynamics Simulations Predict The Pathways via which Pristine Fullerenes Penetrate Bacterial Membranes
}

\begin{tabular}{|r|l|}
\hline Journal: & The Journal of Physical Chemistry \\
\hline Manuscript ID & jp-2016-06615a.R2 \\
\hline Manuscript Type: & Article \\
\hline Date Submitted by the Author: & n/a \\
\hline Complete List of Authors: & $\begin{array}{l}\text { Hsu, Pin; University of Southampton, Chemistry } \\
\text { Jefferies, Damien; University of Southampton, Chemistry } \\
\text { Khalid, Syma; University of Southampton, Chemistry }\end{array}$ \\
\hline
\end{tabular}

SCHOLARONE ${ }^{m}$

Manuscripts 


\title{
Molecular Dynamics Simulations Predict The Pathways via which Pristine Fullerenes Penetrate Bacterial Membranes.
}

\author{
Pin-Chia Hsu, ${ }^{\mathrm{a},}+$ Damien Jefferies ${ }^{\mathrm{a},}+$ and Syma Khalid ${ }^{\mathrm{a}^{*}}$ \\ a. School of Chemistry, University of Southampton, Southampton, SO17 1BJ, UK \\ $\dagger$ These authors contributed equally \\ * to whom correspondence should be addressed at Email: S.Khalid@soton.ac.uk Tel.: \\ +44-2380-594176 Fax: +44-2380-593781
}




\begin{abstract}
Carbon fullerenes are emerging as effective devices for different biomedical applications, including the transportation of nanosized drugs and the extraction of harmful oxidants and radicals. It has been proposed that fullerenes could be used as novel antibacterial agents, given the realization that the nanoparticles can kill pathogenic Gram-negative bacteria. To explore this at the molecular level, we simulated $\mathrm{C}_{60}$ fullerenes with bacterial membranes using the coarse-grain (CG) molecular dynamics (MD) Martini force field. We find that pristine $\mathrm{C}_{60}$ has a limited tendency to penetrate (incomplete core) Re mutant lipopolysaccharide (LPS) leaflets, but the translocation of $\mathrm{C}_{60}$ fullerenes into (complete core) Ra mutant LPS leaflets is not thermodynamically favored. Moreover, we show that the permeability of Re LPS bilayers depends sensitively on system temperature, the charge of ambient ions, and the prevalence of POPE defect domains. The different permeabilities are rationalized in terms of transitory head group pore formation, which underpins the translocation of $\mathrm{C}_{60}$ into the lipid core. The Re LPS lipids readily form transient micropores when they are linked with monovalent cations, or when they are heated to a high temperature. POPE lipids are shown to be particularly adept at forming these transient surface cavities, and their inclusion into Re LPS membranes facilitates the formation of particularly large pores that are tunneled by $C_{60}$ aggregates of significant size ( $5 \mathrm{~nm}$ wide). After inserting into the lipid core, the aggregates dissociate, and the disbanded nanoparticles migrate to the interface between separate POPE and LPS domains, where they weaken the boundaries between the coexisting lipid fractions and thereby promoting lipid mixing.
\end{abstract}




\section{Introduction}

Since their discovery in $1985,{ }^{1}$ carbon fullerenes have been the subject of intense research due to their interesting chemical properties, and for their potential technological and biomedical applications. For example, their excellent electron acceptor abilities make carbon fullerenes suitable components of novel photovoltaic devices. ${ }^{2}$ Diverse biomedical applications have additionally been proposed for fullerenes, which generally use the ability of these caged compounds to entrap small molecules and thus serve as nanosized gene or drug delivery devices. ${ }^{3,4}$ But more recently, fullerenes have been shown to be bactericidal towards Gram-negative bacteria such as Escherichia coli (E. coli), ${ }^{5,6}$ suggesting that fullerenes may find future applications in controlling microbial pathogens. $C_{60}$ nanoparticles seem especially well suited for antibacterial applications compared to metallic nanoparticles or expansive repetitively branched dendrimers, given their small size, which reduces energy barriers to bilayer insertion, and their ability to accommodate diverse surface decoration enabling fine-tuning of particle shape and surface chemistry. ${ }^{7,8}$ As such, work investigating the antibacterial action of fullerenes through the use of experimental techniques has become one of the most topical areas in bionanotechnology. ${ }^{9-11}$

Molecular dynamics (MD) provides an alternative approach for studying the dynamic interaction of fullerenes with cells in molecular-level detail. Previous atomistic simulation studies of bacterial membranes have shown that timescales required to observe penetration into these resilient membranes necessitates the use of coarsegrain (CG) MD. ${ }^{12,13}$ In fact, simulations have shown that the translocation of pristine $\mathrm{C}_{60}$ fullerenes through even the simplest of phospholipid bilayers can occur on a microsecond timescale, which is too demanding for conventional all-atom MD. ${ }^{14-17}$ Here we report CG MD simulations of the interaction of $C_{60}$ nanoparticles with model bacterial membranes. The membrane models are composed of lipopolysaccharide (LPS) and palmitoyloleoylphosphoethanolamine (POPE) lipids. Through this series of CG simulations, we show that $C_{60}$ fullerene translocation depends on specific lipid composition. The fullerenes have a limited tendency to penetrate bacterial 
membranes whose major component is Re mutant LPS i.e. the minimal biologically active structure of endotoxins, ${ }^{18}$ which consists of lipid A and two molecules of 3deoxy-D-manno-2octulosonic acid (KDO), but the translocation of $\mathrm{C}_{60}$ fullerenes into bilayers whose major component is Ra LPS, which contains additional peripheral head group sugars (Figure 1), ${ }^{19}$ is an energetically unfavorable process. Moreover, we show that $\mathrm{C}_{60}$ fullerenes more frequently penetrate Re LPS bilayers when they are loaded with ions of low charge, when they are heated to a high temperature, or when they incorporate small defect domains of POPE. The submerged fullerenes tend to migrate to the interface between coexisting lipid fractions, where they promote lipid mixing by reducing the line tension between the separate bilayer domains. The differing permeabilities of the Re LPS bilayers are rationalized in terms of their translocation mechanism, which involves the localized expansion of lipids adjacent to $\mathrm{C}_{60}$ molecules at the membrane interface.

\section{Experimental}

\section{CG models of LPS}

The models of LPS (at the Re and Ra levels) are similar to those reported by Ma et al. and Van Oosten et al. ${ }^{20,21}$ Small modifications were made to improve the accuracy of the models based on our own united-atom (UA) simulations. The mappings used for the CG models, and the chemical structure of the UA models from which they were parameterized are shown in Figure 1. 

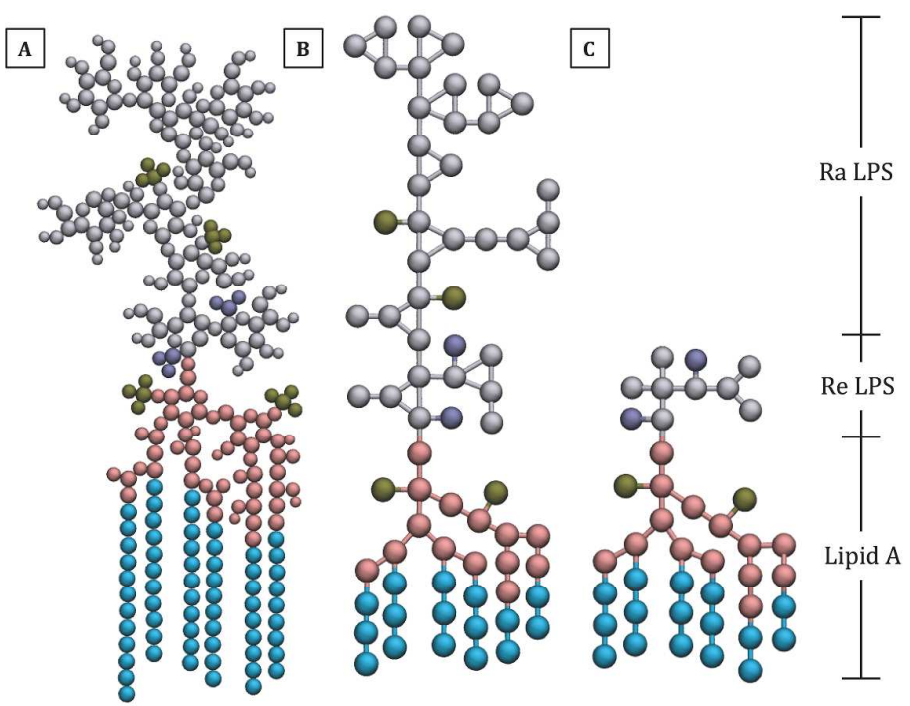

Figure 1: CG mapping schemes for Re and Ra LPS (A) The UA model for Ra LPS is shown alongside the CG model for (B) Ra LPS, and (C) Re LPS. Carbon tails are colored cyan, phosphate groups are colored brown, carboxylate groups are colored dark blue, glucosamine and glycerol groups are colored pink, and the remaining core saccharide sections are colored silver.

We analyzed lipid organization in the comparable UA and CG E. coli bacterial membranes by computing one-dimensional partial mass densities of lipid components along the bilayer normal (Figure 2). The peaks of the carbon tails, inner core sugars, and glucosamine and glycerol groups are equivalent to within $0.3 \mathrm{~nm}$ for both $\operatorname{Re}$ and Ra LPS, the outer core sugar peaks differ by $\sim 0.4 \mathrm{~nm}$. These simulations were performed with periodic boundary conditions throughout, ensuring that each lipid leaflet was in contact with similar solvent. The partial densities are discussed in terms of the Martini treatment of ions in more detail in the SI. Full details of the parameterization and validation of the CG models of LPS used here are provided in the supplementary information (Figure S1-S2, Table S1S9). 

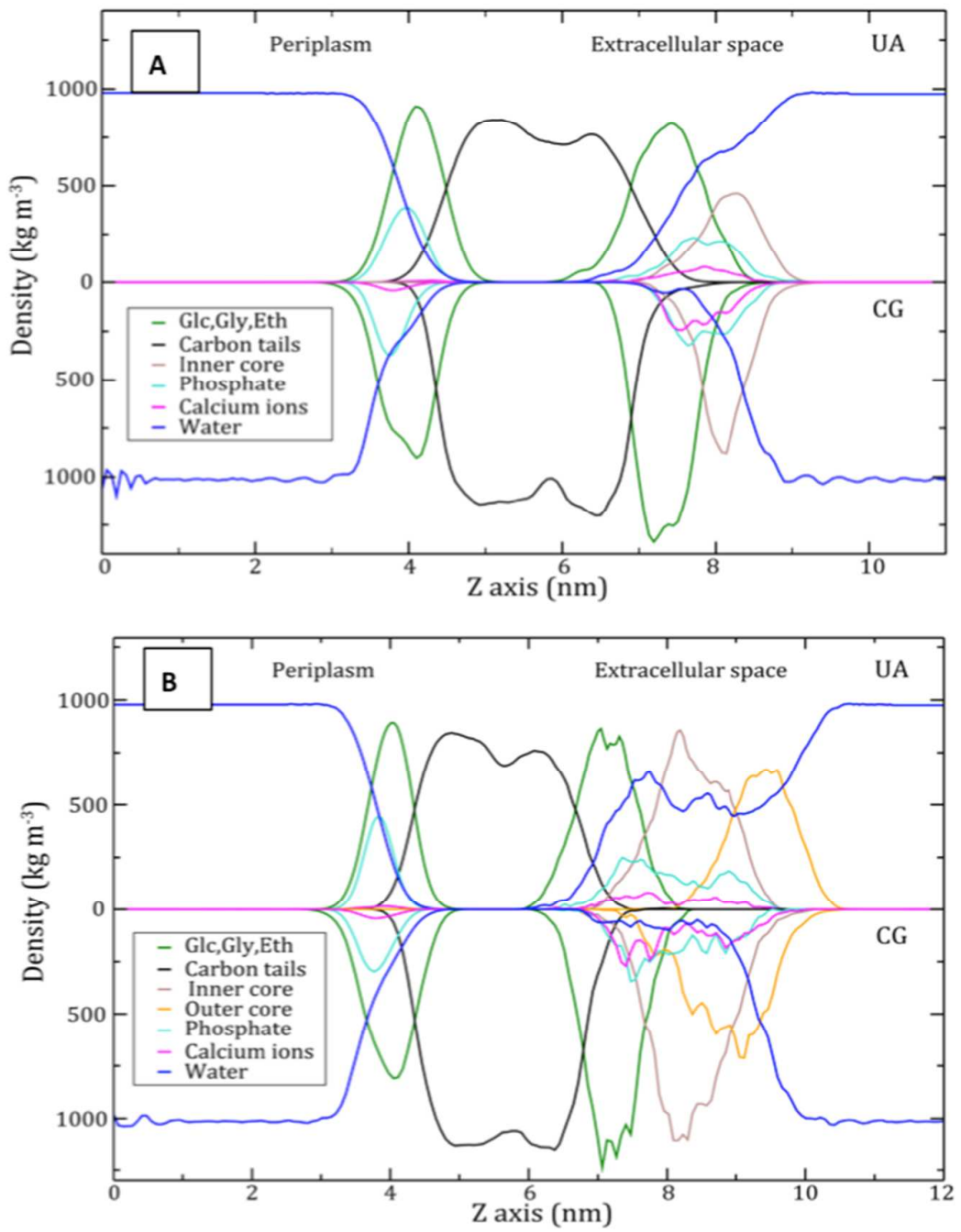

Figure 2: Comparative partial mass density plots for $E$. coli outer membranes. $E$. coli membranes were simulated at $310 \mathrm{~K}$ using CG and UA representations. The first membrane was built with a leaflet of (A) Re LPS, while the second membrane contained a leaflet of (B) Ra LPS instead. Carbon tails are represented by black lines, glucosamine, glycerol and ethanolamine groups (Glc,Gly,Eth) are represented by green lines, phosphate groups are represented by turquoise lines, calcium ions are represented by pink lines, inner core sugars are represented by brown lines, outer core sugars are represented by orange lines, and water is represented by blue lines. The partial mass densities are measured along the bilayer normal ( $Z$ axis). 


\section{CG simulation systems and protocols}

The CG membranes were placed in the center of a periodic box and immersed in standard Martini CG water and ions. The systems were energy minimized with the steepest decent algorithm, and subsequently simulated at constant temperature and pressure. Model membranes were composed of (i) only LPS and (ii) LPS with small defect domains of POPE. POPE is the most abundant phospholipid in Gram-negative membranes and has been found in small concentrations in the outermost LPS leaflets as a stress response for LPS mutants of complete core saccharide section, and among incomplete core LPS mutants under standard conditions. ${ }^{22,23}$ In each instance, the membranes were solvated with CG water, anions $\left(\mathrm{Cl}^{-}\right)$and cations $\left(\mathrm{Ca}^{2+}\right.$ or $\mathrm{Na}^{+}$) and subsequently simulated for $5 \mu \mathrm{s}$, to converge the lipid properties. Divalent cations are essential for retaining the integrity of LPS-containing leaflet as they cross-link the head groups. ${ }^{24}$ We used a symmetric bilayer instead of an asymmetric model of the outer membrane purely for reasons of computational pragmatism. We did not want the $\mathrm{C}_{60}$ to cross the periodic boundaries and insert via the more penetrable inner leaflet given that our main focus is the interaction with the LPS-containing leaflet. The temperature was maintained at either 310 or $325 \mathrm{~K}$ using the velocity-rescaling thermostat ${ }^{25}$ with a relaxation time of $4 \mathrm{ps}$, while the pressure was maintained at 1 bar through semi-isotropic coordinate scaling with the Parrinello-Rahman barostat ${ }^{26,27}$ and a time constant of 12 ps. The higher temperature of $325 \mathrm{~K}$ was used to enable greater coverage of the phase space, rather than for biological relevance. The non-bonded interactions were cut off at a distance of $1.2 \mathrm{~nm}$. The Lennard-Jones, and Coulomb potentials were shifted from 0.9 and 0.0 $\mathrm{nm}$ to the cutoff distance, respectively. The equations of motion were integrated with a $10 \mathrm{fs}$ time step. Following this, the bilayers were brought into contact with different concentrations of $\mathrm{CG}_{60}$ fullerenes to provide a comprehensive account of how monomeric and clustered $\mathrm{C}_{60}$ fullerenes interact with model bacterial membranes. The $\mathrm{CG} \mathrm{C}_{60}$ fullerenes were described with a parameter set that was optimized to reproduce partitioning free energies for fullerenes between different organic solvents. ${ }^{28}$ The simulations were performed with the GROMACS simulation package (version 4.5.5), and the Martini force field (version 2.2). Molecular graphic 
images and trajectories were visualized with Visual Molecular Dynamics (VMD) software. $^{29}$

\section{Analyses of the unrestrained $C_{60}$ fullerene simulations}

Contact analysis was performed with the g_mindist utility of GROMACS using a 0.6 $\mathrm{nm}$ cutoff distance. The position of molecules along the bilayer normal was determined with the g_traj function. Two-dimensional partial mass density landscapes were generated with a modified version of the g_density tool. ${ }^{30}$

\section{Umbrella sampling simulations}

$\mathrm{C}_{60}$ monomers were restrained at distances from the center of homogeneous membranes using a harmonic potential, a force constant of $1000 \mathrm{KJ} \mathrm{mol}^{-1} \mathrm{~nm}^{-2}$ was used for the biasing potential in the direction of the bilayer normal. The distance between the fullerene and the center of the bilayer was decreased from a distance of $5.2 \mathrm{~nm}$ to $0 \mathrm{~nm}$, using $0.2 \mathrm{~nm}$ increments. Each umbrella sampling simulation was $1 \mu \mathrm{s}$ long; the simulation temperature was $310 \mathrm{~K}$ throughout. All other simulation parameters were set to match those of the unrestrained MD simulations.

\section{Results and Discussion}

\section{Unrestrained MD simulations}

To understand how fullerenes affect the outer membrane of Gram-negative bacteria, we performed simulations to understand how pristine $C_{60}$ nanoparticles interact with pure membranes of LPS, and membranes of LPS with small defect domains of POPE. In each instance, the membranes had equivalent lipid distributions in either leaflet, to ensure that the initial $\mathrm{C}_{60}$-membrane interaction remained much the same if the fullerenes happened to cross a boundary of the periodic box. The series of simulations covered a range of different fullerene concentrations, providing a comprehensive account of how both monomeric, and clustered fullerenes interact with bacterial LPS. The lowest $\mathrm{C}_{60}$ concentration was $0.01 \mathrm{mM}$, and the highest was $1.13 \mathrm{mM}$; experimental studies suggest that $\mathrm{C}_{60}$ fullerenes are antibacterial towards 
E. coli at concentrations as low as $0.06 \mathrm{mM} .^{5}$ Tables S10 and Table S11 provide additional details for these simulations, including the numbers of $C_{60}$ fullerenes per periodic box, structural properties for the lipid bilayers, and the numbers of $\mathrm{C}_{60}$ fullerenes that bypassed the phosphate boundary during the MD simulations.

\section{Pure LPS membranes}

The first set of simulations explored how individual fullerenes interact with membranes containing only Re or Ra LPS. The simulations were performed at both CG and UA levels of detail, to provide a comparison for the different resolution levels (details of the UA simulation conditions are provided in the supplementary information). In the first part of the $\mathrm{C}_{60}$ monomer simulations, the fullerenes traversed the extracellular space (modeled as water and ions), and subsequently latched onto the peripheral saccharide sections of LPS. From there on, the fullerenes remained at the water-core sugar interface, displaying little lateral or vertical movement. For the Re LPS bilayers, the fullerenes maintained a height of $0.92 \pm 0.11$ $\mathrm{nm}$ above the phosphate interface in the UA simulation, and $1.00 \pm 0.06 \mathrm{~nm}$ in the comparative CG system (sampling the last 100 ns of simulation time). In comparison, the $\mathrm{C}_{60}$ monomers maintained heights of $3.32 \pm 0.16 \mathrm{~nm}$ in the UA simulation system with Ra LPS, and $2.19 \pm 0.04 \mathrm{~nm}$ for the analogous CG setup (Figure S3). The mismatch in data for the Ra LPS system reveals the major deficiency of our CG LPS parameterization and its implication for simulations with $\mathrm{C}_{60}$ : the height of the peripheral core saccharide sections differs between the UA and CG membranes, making the initial interactions between $C_{60}$ and Ra LPS different for the two resolution levels.

The low permeability of the LPS bilayers to monomeric fullerenes stands in stark contrast to the efficient solubilizing properties of most phospholipid membranes, which readily dissolve large amounts of $C_{60}$ and $C_{70}$ fullerenes. ${ }^{31-33}$ This seemingly anomalous behavior could be attributed to the presence of densely packed core sugars in the LPS head group region, or alternatively to differences in the hydrophobic core of LPS and phospholipid bilayers. In either case, the translocation of $\mathrm{C}_{60}$ fullerenes into the bilayers should occur more frequently at higher 
temperatures. To test if an increased simulation temperature would enable $\mathrm{C}_{60}$ translocation we repeated the $\mathrm{C}_{60}$ monomer simulations at $325 \mathrm{~K}$. At this temperature, the Re LPS lipids frequently formed small cavities at the phosphate interface whose size was comparable to that of the $C_{60}$ fullerenes. As the fullerenes came into contact with these transitory micropores, they slipped past the hydrophilic head barrier, and thereby accessed the lipid core. Thereafter, the nanoparticles dispersed among the encompassing lipid tails, maintaining a distance of approximately $1 \mathrm{~nm}$ from the bilayer center (Figure S4). This mimics the positions that fullerenes occupy in generic palmitoyloleoylphosphatidylcholine (POPC) and dipalmitoylphosphatidylcholine (DPPC) lipid bilayers, ${ }^{34,35}$ this similarity in $\mathrm{C}_{60}$ trajectories has been ascribed to higher acyl chain densities in this part of the bilayer. In contrast, the Ra LPS membrane permeability was seemingly unchanged: the $\mathrm{C}_{60}$ fullerenes did not penetrate the core sugar envelope to access the hydrophobic core regardless of system temperature.

For the high concentration simulations $\left(0.47\right.$ and $\left.1.13 \mathrm{mM} \mathrm{C}_{60}\right)$ the nanoparticles aggregated in the extracellular space to form globular clusters, that match nanosized $\mathrm{C}_{60}$ crystals observed in transmission electron microscopy studies. ${ }^{36}$ We explored the clustering of the nanoparticles in terms of size and lifetime of $C_{60}$ aggregates, the analysis was performed by measuring the distances between $C_{60}$ fullerenes during the CGMD simulations. Whenever this separation distance was smaller than $1.3 \mathrm{~nm}$, the molecules were defined as clustered. The selected cutoff distance $(1.3 \mathrm{~nm})$ is comparable to maximums in dimerization free energy profiles for $\mathrm{CG}_{60}$ models in different polar and apolar solvents and thus seems a sensible metric for aggregation. ${ }^{37}$ Our results revealed that $C_{60}$ fullerenes form structures at least $5 \mathrm{~nm}$ wide, within the first 400 ns of simulation time (Figure 3). Once clustered, the fullerenes remained in an aggregated state as they latched onto the peripheral saccharide sections of Re and Ra LPS. But despite their large size, the $\mathrm{C}_{60}$ aggregates had negligible effect on the dynamic properties of the membrane. Lipid surface area changed by less than $0.02 \mathrm{~nm}^{2}$, membrane thickness changed by less than $0.2 \mathrm{~nm}$, and there was only a minute modification of lipid order (Table S10). The small perturbation of bilayer structural parameters in the various fullerene simulations 
suggests that direct mechanical damage (e.g. bilayer rupture, or the formation of pores) is not responsible for the antibacterial effects of $C_{60}$.
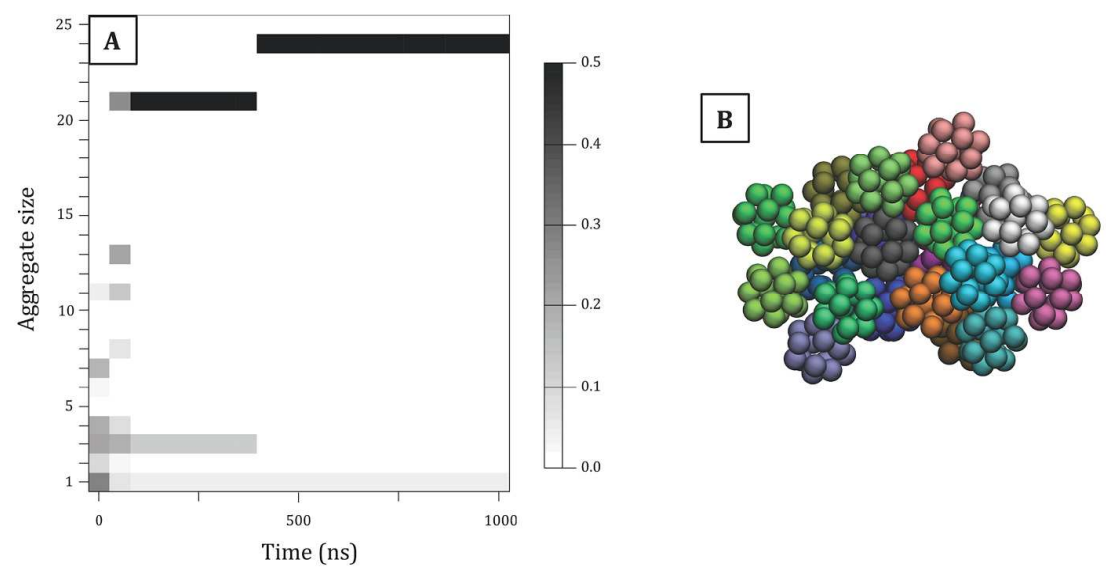

Figure 3: Clustering of $C_{60}$ in a representative $C G$ simulation (A) The clustering of $C_{60}$ is plotted as a function of time during the first $1 \mu$ s of a CG simulation. The grayscale in each cell represents the fraction of $C_{60}$ involved in an aggregate of a given size ( $y$ axis) at a given time ( $x$ axis). The $\mathrm{C}_{60}$ concentration was $1.13 \mathrm{mM}$. (B) Snapshot of the 24-unit $\mathrm{C}_{60}$ cluster formed toward the end of this initial period of aggregation.

\section{Simulations with sodium ions}

Given that LPS leaflets display markedly different properties in the presence of different cations, we explored how fullerenes would interact with LPS membranes in the presence of (standard CG Martini) monovalent sodium ions (in place of the calcium ions used before). Similar to results from atomistic simulation and experimental studies, the LPS leaflets seemed more penetrable when they were loaded with ions of low charge. ${ }^{38,39}$ The monovalent cations were less able to screen the repulsive electrostatic interactions between the neighboring molecules of LPS, which lead to increased lateral expansion within the bilayer plane. The Re LPS area per lipid increased from a value of $1.58 \pm 0.00005 \mathrm{~nm}^{2}$ to a value of $1.70 \pm 0.0001$ $\mathrm{nm}^{2}$ at $310 \mathrm{~K}$, while the membrane thinned from a thickness of $3.39 \pm 0.0001 \mathrm{~nm}$ to $3.25 \pm 0.0002 \mathrm{~nm}$. Evidently, the Re LPS bilayers present a less formidable barrier in the presence of monovalent cations, and as a consequence, the $\mathrm{C}_{60}$ fullerenes more readily entered the Re LPS bilayer core. This is evident for the simulations performed at the lower, biologically relevant temperature of $310 \mathrm{~K}$ (Table S11), where $\mathrm{C}_{60}$ 
fullerenes successfully bypassed the LPS head group barrier, to access the lipid core. The translocation occurred as $\mathrm{C}_{60}$ monomers came into contact with transient micropores. Two-dimensional voronoi tessellations of the membrane surface show that the micropores were the product of localized lateral expansion of the Re LPS bilayer. One of these micropores was formed as three LPS lipids expanded within the bilayer plane to increase the local area per lipid from a value of $1.70 \pm 0.0001 \mathrm{~nm}^{2}$ to $2.02 \mathrm{~nm}^{2}$, and in so doing, temporarily establish a cavity in the bilayer surface (Figure 4).
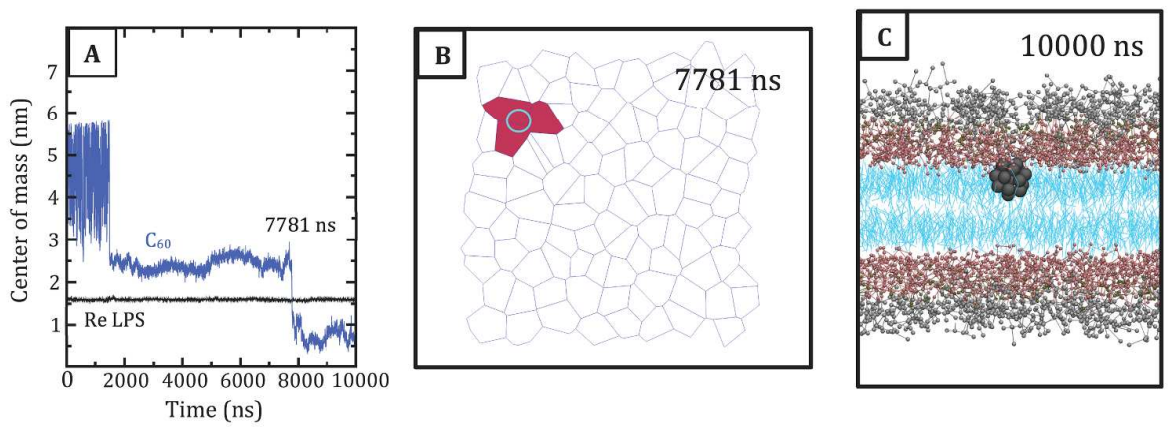

Figure 4: The effect of sodium ions on Re LPS bilayer permeability. (A) Position of the $\mathrm{C}_{60}$ monomer along the bilayer normal, relative to the Re LPS bilayer center. The blue line depicts the center of mass coordinates for the $C_{60}$ monomer from the bilayer center, while the black line shows the average position of LPS boundary phosphate groups in a single leaflet of the bilayer. The simulation was performed at $310 \mathrm{~K}$, with a single $\mathrm{C}_{60}$ fullerene. (B) Two-dimensional voronoi tessellation of the $\mathrm{Re}$ LPS surface as the $\mathrm{C}_{60}$ fullerene bypasses the phosphate barrier at $7781 \mathrm{~ns}$. Each cell represents a single Re LPS head group. The red cells correspond to the LPS head groups adjacent to the $\mathrm{C}_{60}$ monomer. The cyan circle shows the position of the $\mathrm{C}_{60}$ monomer during the head group tunneling event. (C) Side-view snapshot for the $C_{60}$ monomer after it bypassed the phosphate interface to settle in the lipid core.

The formation of transient micropores via localized lateral lipid expansion facilitates the insertion of monomeric $\mathrm{C}_{60}$ fullerenes into the Re LPS bilayer core, but larger multimeric clusters were unable to bypass the phosphate boundary. As a 
consequence, there was only partial embedding of $C_{60}$ fullerenes into the Re LPS membrane when the fullerene concentration was increased from $0.02 \mathrm{mM}$, to 0.35 $\mathrm{mM}$. At this concentration, most of the fullerene monomers had tunneled through hydrophobic pores to access the lipid core, while the larger globular clusters interacted with the peripheral LPS saccharide domain.

For the Ra LPS bilayer, the area per lipid increased from $1.83 \pm 0.00004 \mathrm{~nm}^{2}$ at $310 \mathrm{~K}$, to $1.95 \pm 0.00004 \mathrm{~nm}^{2}$ as the calcium ions were exchanged for less polar sodium ions. But despite the changes in the structural properties of the membrane, the $C_{60}$ fullerenes were unable to pass through the phosphate interface.

\section{Mixed lipid membranes}

Given that the endotoxin (LPS) leaflet of bacterial outer membranes can incorporate small phospholipid defect domains in vivo, ${ }^{40-43}$ we explored the possibility that $\mathrm{C}_{60}$ preferentially interacts with these lipids over (both Re and Ra) LPS. We replaced approximately $25 \%$ of the LPS membrane surface with small clusters of POPE lipids. The clusters covered a range of different sizes ranging from 10 to 25 POPE lipids. Similar to the results from experimental studies, ${ }^{22-23,44}$ the POPE lipids tended to separate from the encompassing LPS, dividing the membrane into two distinct sections. After the dual component membranes had divided into distinct fractions, they were brought into contact with a range of different fullerene concentrations.

The incorporation of POPE lipids made the membranes more penetrable to the $\mathrm{C}_{60}$ nanoparticles. At low concentrations, fullerene monomers accessed the membrane cores more frequently (per simulation) as they passed through the POPE headgroups. After inserting into the lipid core, the monomers traversed the acyl tail section, staying at least $0.5 \mathrm{~nm}$ from the bilayer center. 

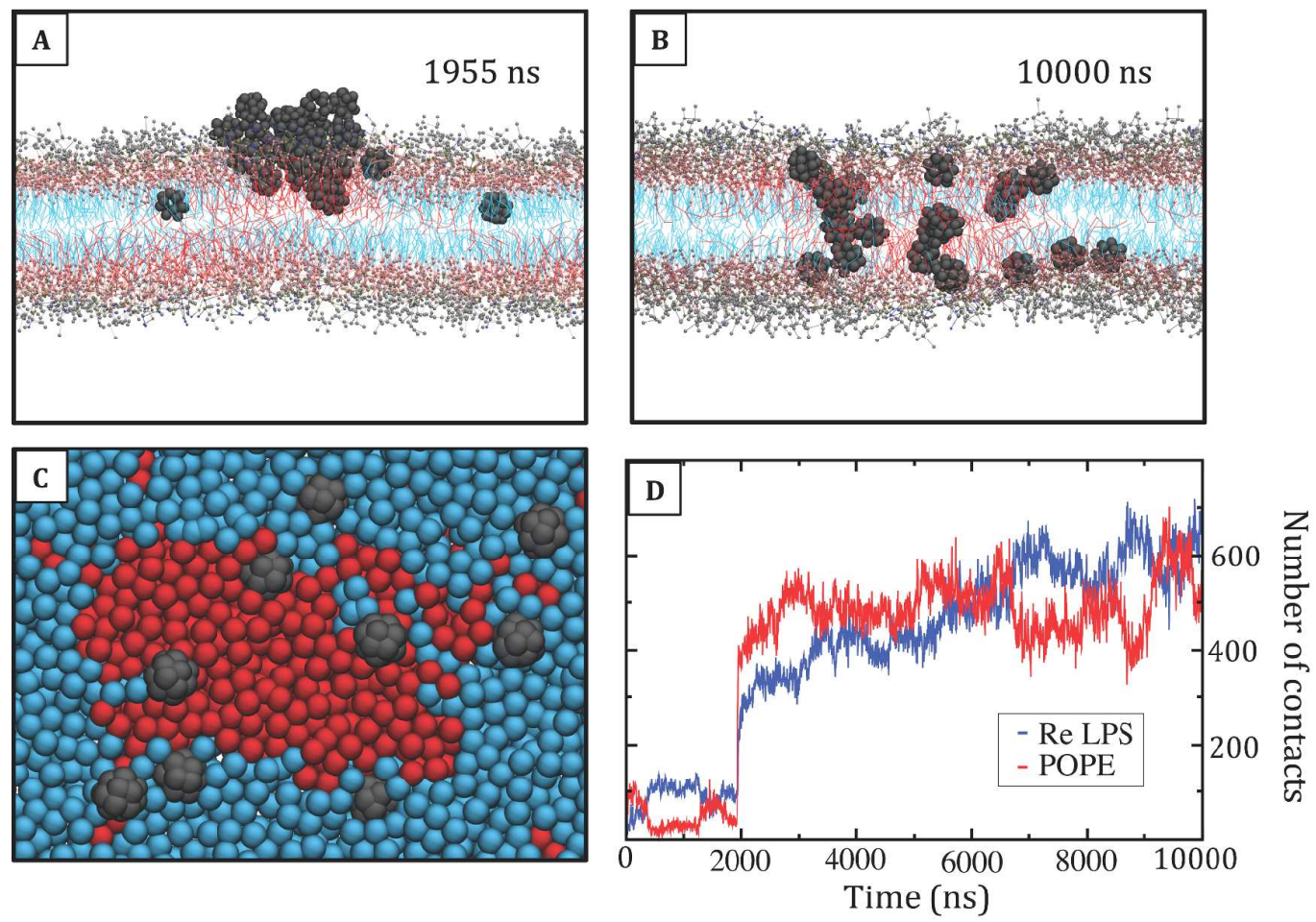

Figure 5: Migration of $C_{60}$ fullerenes to the POPE-Re LPS lipid interface. (A) The $C_{60}$ cluster migrates to the domain of POPE, and slips past the hydrophilic head groups to access the lipid core. (B) The cluster disaggregates, and the disbanded fullerenes drift to the interface between the separate POPE and LPS domains. The Re LPS are colored as before, and the POPE lipid tails are colored red. (C) Top-view snapshot of the disbanded fullerenes at the POPE-Re LPS lipid interface. The lipid head groups are omitted for clarity. (D) The number of contacts between the $\mathrm{C}_{60}$ fullerenes and the (Re LPS and POPE) acyl tails is plotted as a function of simulation time. The red line shows the number of contacts between the fullerenes and POPE lipid tails, while the blue shows the number of contacts between the fullerenes and the Re LPS lipid tails. The relative number of contacts was determined using a $0.6 \mathrm{~nm}$ cutoff distance. 
For the high concentration simulations $(0.37 \mathrm{mM}$ and $0.66 \mathrm{mM})$, the nanosized $\mathrm{C}_{60}$ clusters were likewise capable of penetrating the multicomponent membranes. Initially, the fullerene monomers coalesced to form globular clusters within the first 300 ns of simulation time. After that, the $C_{60}$ aggregates migrated to the POPE lipids and passed through their head groups, or alternatively they remained atop the peripheral saccharide section of LPS (Figure $5 A, B$ ). We explored how this insertion event took place through the use of two-dimensional projections of phosphate and choline mass densities, voronoi tessellations of LPS and POPE head group atoms, and separation distance calculations. By partitioning the membrane surface into distinct domains based on local area per lipid and local particle densities, we resolved the translocation process. At the moment of insertion, approximately 10 POPE headgroups moved apart to create a micropore whose shape and surface area matched those of the $C_{60}$ aggregate (Figure 6). The POPE area per lipid value approached $2.0 \mathrm{~nm}^{2}$ during this time (Figure 6F), while the POPE width increased by approximately $10 \%$. These local deforming effects were gradually suppressed as the cluster subsequently broke apart, and the disconnected $\mathrm{C}_{60}$ monomers dispersed among the surrounding lipid tails. Similar disaggregation behavior has been noted in phospholipid bilayers, and rationalized as the result of high carbon atom densities, and the perturbation of chain packing by the fullerene clusters. ${ }^{45}$ 

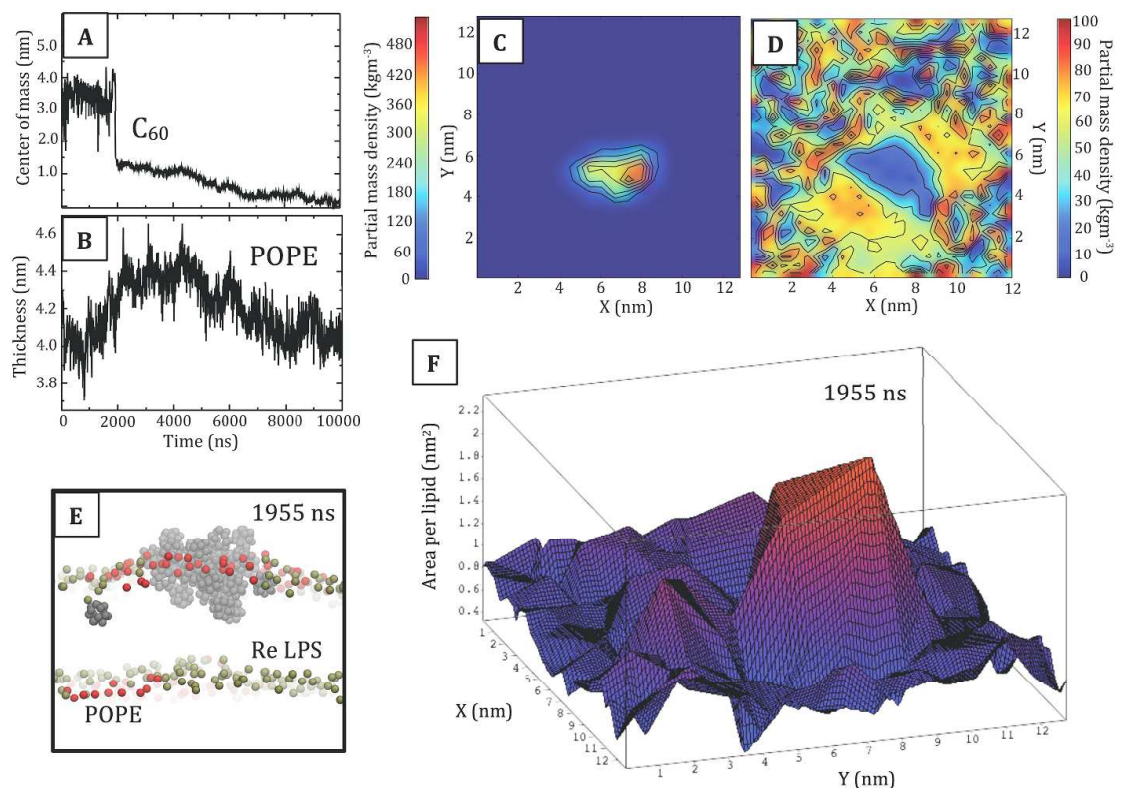

Figure 6: Translocation of a $\mathrm{C}_{60}$ cluster across the water-lipid interface. (A) Average center of mass coordinates for $\mathrm{C}_{60}$ fullerenes along the bilayer normal (relative to the bilayer center). (B) Average thickness of the POPE lipids plotted against simulation time; thickness was defined as the distance between POPE phosphate head groups and the bilayer center. (C) Two-dimensional partial mass density for $\mathrm{C}_{60}$ fullerenes comprising the $\mathrm{C}_{60}$ cluster, during the bilayer insertion event (1955 ns). (D) Partial mass densities for Re LPS phosphate and POPE choline head groups during fullerene cluster insertion (1955 ns). The POPE head groups move apart to create a transient micropore whose shape and projected surface area match those of the $\mathrm{C}_{60}$ cluster. (E) Side-view snapshot of the $\mathrm{C}_{60}$ cluster traversing the phosphate interface, the Re LPS phosphate groups are colored brown, the POPE phosphate groups are colored red. All other membrane components are omitted for clarity. (F) Three-dimensional projection of area per lipid values for the membrane leaflet that the $\mathrm{C}_{60}$ fullerenes pass through. The tessellation was performed for the membrane surface at 1955 ns. Red color represents high area per lipid while blue color represents low area per lipid.

Towards the end of the CG simulations, the disconnected monomers accumulated at the interfacial regions between the Re LPS and POPE domains. This was discerned by 
calculating contact numbers for the $\mathrm{C}_{60}$ fullerenes and the carbon tails of the dualcomponent bilayer (using a $0.6 \mathrm{~nm}$ cutoff distance). The number of contacts between the POPE lipid tails and $C_{60}$ fluctuated about an average value once the fullerenes had passed through the phosphate interface, while the number of contacts between the Re LPS tails and the fullerenes initially increased, before converging to a definite value. The results indicate that the $C_{60}$ fullerenes were migrating towards the interfacial regions of the multicomponent membranes during the course of the MD simulation. The behavior is reminiscent of linactants, ${ }^{46-49}$ which tend to collect at the interface between liquid-ordered and liquid-disordered domains, and promote lipid mixing by destabilizing the phase boundary. To explore the possibility that the embedded fullerenes were affecting the boundaries between the separate domains of Re LPS and POPE, we calculated how lipid properties changed in the presence of the fullerenes. In particular, we focused on the simulations with complete immersion of all available $\mathrm{C}_{60}$ fullerenes in the bilayer core, to remove any extraneous influence of fullerene clusters binding to the peripheral saccharide section of LPS. We found that the dispersion of $\mathrm{C}_{60}$ fullerenes in the lipid core lead to greater homogeneity of lipid properties for the separate LPS and POPE domains. There was a general reduction in the mismatch of bilayer thickness, lipid order, and area per lipid, which lead to a reduction in the line tension (boundary free energy) between the coexisting lipid fractions. For example, the thickness mismatch decreased by $0.2 \mathrm{~nm}$ when 25 fullerenes were introduced into the core of the membrane with Re LPS and POPE (Figure 7). The changes in lipid properties suggest that the fullerenes were promoting lipid mixing and encouraging the formation of a more uniform membrane. 

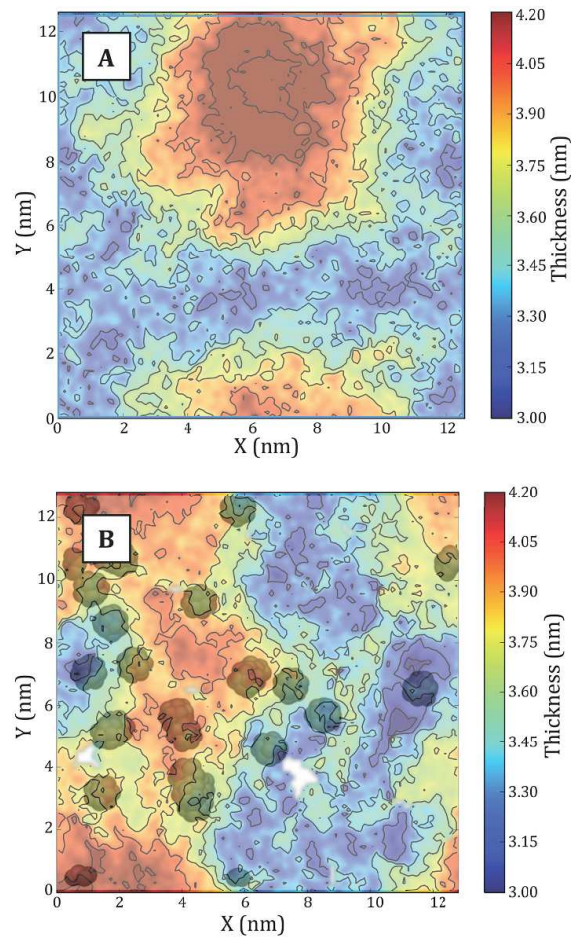

Figure 7: $\mathrm{C}_{60}$ fullerenes promote lipid mixing in the multicomponent membrane. After the $\mathrm{C}_{60}$ fullerenes had entered the bilayer core, they migrated to the boundaries between the coexisting POPE and Re LPS domains. The fullerenes then reduced the line tension between the coexisting lipid fractions by minimizing structural mismatch. The figures depict the bilayer thickness as a two-dimensional landscape for the membrane (A) without any $\mathrm{C}_{60}$ fullerenes dissolved in the lipid core, and (B) with $25 \mathrm{C}_{60}$ fullerenes dispersed among the lipid tails. The final $\mathrm{C}_{60}$ particle positions are depicted above the thickness landscape as translucent black spheres (for reference).

\section{Free energy profiles}

Given the diverse interactions between $C_{60}$ fullerenes and the LPS bilayers observed in the unrestrained MD simulations, we calculated potential of mean force (PMF) profiles to better understand the different bilayer permeabilities. Single $C_{60}$ fullerenes were restrained at different distances from homogeneous bilayers of Re LPS, Ra LPS, and POPE in the presence of either monovalent sodium ions $\left(\mathrm{Na}^{+}\right)$, or divalent calcium ions $\left(\mathrm{Ca}^{2+}\right)$. The weighted histogram analysis method was 
subsequently utilized to produce a collection of PMF profiles, which are shown in Figure 8. The analysis reveals that $\mathrm{C}_{60}$ translocation is thermodynamically favored for POPE and Re LPS bilayers regardless of ambient ion type, but the translocation of $C_{60}$ fullerenes into Ra LPS bilayers is energetically unfavorable. The data show that increasing the length of the LPS core sugar section can profoundly influence the thermodynamics of $\mathrm{C}_{60}$ translocation, and likewise suggests that phospholipid defect domains can make LPS containing bilayers permeable to $C_{60}$ fullerenes. Moreover, the PMF profiles help to clarify fullerene trajectories in the Re LPS and POPE lipid core. The PMF profiles have (global) free energy minima at distances of approximately 0.5 and $1.5 \mathrm{~nm}$ from the bilayer center for the Re LPS and POPE bilayers, respectively. These energy minima reveal that it is more favorable for the $\mathrm{C}_{60}$ nanoparticles to settle among the high density Re LPS lipid tails than the bilayer center, explaining the tendency for $\mathrm{C}_{60}$ fullerenes to avoid the bilayer center in the unrestrained MD simulations.

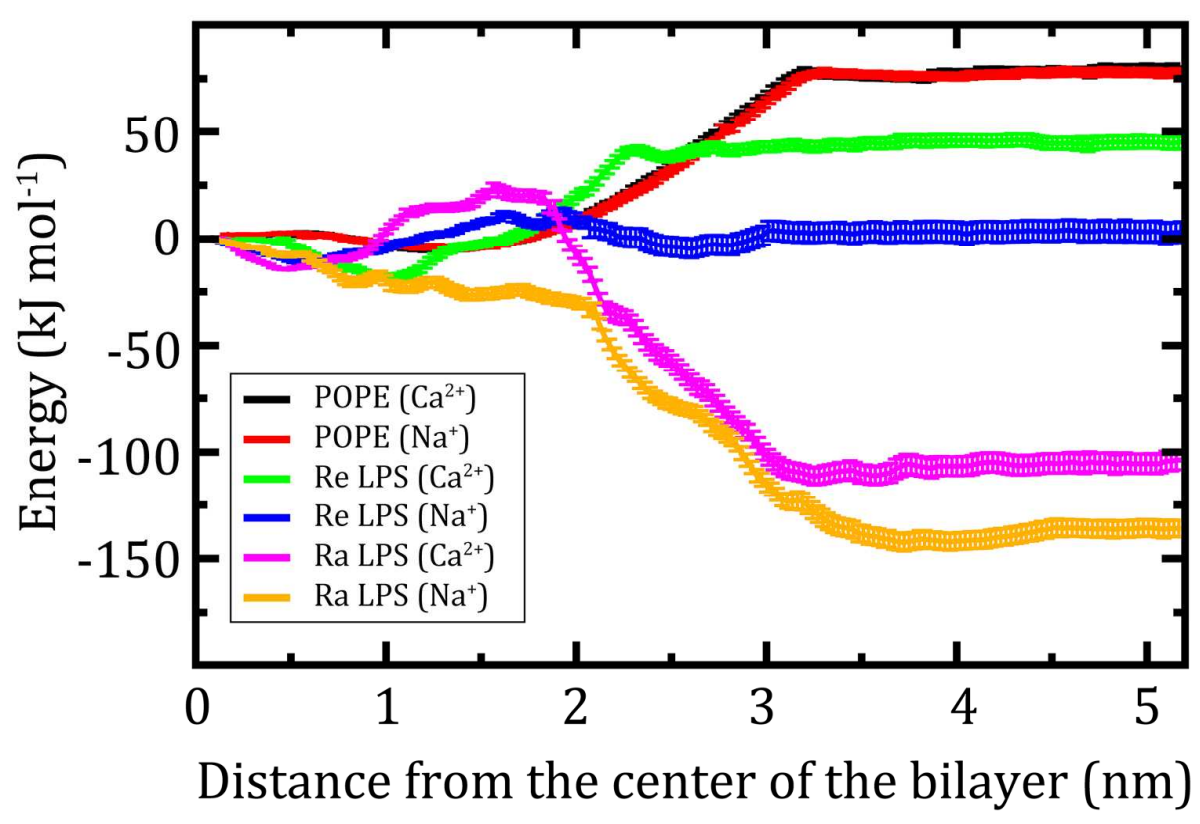

Figure 8: $P M F$ profiles for $\mathrm{C}_{60}$ fullerene as a function of distance from the center of homogeneous lipid bilayers. PMF profiles for $\mathrm{C}_{60}$ fullerene as a function of distance from the bilayer center. The red line shows the data for the POPE bilayer with $\mathrm{Na}^{+}$ 
ions, the black line shows the data for the POPE bilayer with $\mathrm{Ca}^{2+}$, the green line shows the data for the Re LPS bilayer with $\mathrm{Ca}^{2+}$ ions, the blue line shows the data for the Re LPS bilayer with $\mathrm{Na}^{+}$, the pink line shows the data for the Ra LPS bilayer with $\mathrm{Ca}^{2+}$ ions, and the orange line shows the data for the Ra LPS bilayer with $\mathrm{Na}^{+}$ions. Each umbrella sampling simulation was $1 \mu$ s long; the simulation temperature was $310 \mathrm{~K}$; the umbrella sampling windows were separated by distances of $0.2 \mathrm{~nm}$. The free energy was set to zero at the bilayer center.

\section{Conclusion}

A series of $C G$ simulations was performed to better understand the interaction of $C_{60}$ fullerenes with the outer membrane of $E$. coli. We found that pristine $C_{60}$ has a limited tendency to penetrate homogeneous bilayers of (incomplete core saccharide) Re LPS, but the translocation of $C_{60}$ fullerenes into homogeneous (complete core saccharide) Ra LPS bilayers is an energetically unfavorable process. Secondly, we have shown that the permeability of Re LPS bilayers depends sensitively on the charge of ambient ions, on the system temperature, and on the prevalence of phospholipid defect domains (specifically POPE lipids) among the Re LPS lipids. The differing permeabilities were rationalized in terms of transitory pore formation at the phosphate interface, which was driven by localized lipid head group expansion. The Re LPS molecules expanded readily to form large transient micropores when they were interlinked with monovalent (rather than divalent) cations, or when they were heated to a higher temperature, enabling the $C_{60}$ fullerenes to tunnel the phosphate interface more effectively. The POPE lipids were most effective at forming large transient micropores, and their inclusion enabled the formation of the largest transitory cavities that were tunneled by $\mathrm{C}_{60}$ fullerenes both in their monomeric, and clustered form.

When inside the lipid core, the fullerenes behaved similarly to linactants: the fullerenes moved to the interface between the separate POPE and LPS domains, where they reduced the boundary free energy between the coexisting lipid fractions 
by minimizing structural mismatch. ${ }^{50}$ This reduction in the membrane line tension could have important consequences for membrane lateral organization on largerscales, which is pertinent to various cellular processes such as signaling and protein trafficking.

In conclusion, we have demonstrated that the interaction of $C_{60}$ fullerenes with the E. coli outer membranes depends sensitively on ambient temperature; on the length of the LPS core saccharide section; on the prevalence of phospholipid defect domains and the charge of the ions that interlink LPS phosphate groups. The incorporation of even small concentrations of phospholipids, single unit changes in the charge density of stabilizing counterions, or minor modifications of the ambient temperature $(\Delta T=15 \mathrm{~K})$, can profoundly affect how fullerenes interact with bilayers that contain LPS, and where they settle within the membrane. More interestingly, the extension of the LPS core sugar section can change $C_{60}$ fullerene translocation from being an energetically favorable process, to being energetically unfavorable. The results help to explain why seemingly contradictory toxicological evaluations have been obtained for $C_{60}$ and its different derivatives. ${ }^{51-54}$ The mismatch may be ascribed to small differences in temperature, ambient ion concentrations, LPS core sugar length, or the prevalence of phospholipid defect domains leading to significantly different interactions between the $C_{60}$ fullerenes and the Gram-negative outer membranes. We have provided a comprehensive account of how fullerenes bind to, and interact with LPS lipids when they are incorporated into membranes. The molecular level insights from our simulations provide a rational starting point for modifying fullerenes to increase their effectiveness as antibacterial agents.

\section{ASSOCIATED CONTENT}

\section{Supporting Information}

Four figures and eleven tables to validate the LPS parameters and provide additional simulation data.

\section{AUTHOR INFORMATION}




\section{Corresponding author}

*Email: S.Khalid@soton.ac.uk. Tel.: +44-2380-594176. Fax: +44-2380593781.

\section{Acknowledgment}

This project made use of time on ARCHER granted via the UK High-End Computing Consortium for Biomolecular Simulation, HECBioSim (http://hecbiosim.ac.uk), supported by EPSRC (grant no. EP/L000253/1). Computer code was developed was James Graham.

\section{References}

1 Kroto, H. W.; Heath, J. R.; O’brien, S. C.; Curl, R. F.; Smalley, R. E. C60: Buckminsterfullerene Nature 1985, 318, 162-163.

2 Günes, S.; Neugebauer, H.; Sariciftci, N. S. Conjugated Polymer-Based Organic Solar Cells Chem. Rev. 2007, 107, 1324-1338.

3 Mendes, R. G.; Bachmatiuk A.; Büchner, B.; Cuniberti, G.; Rümmeli, M. H. Carbon Nanostructures as Multi-Functional Drug Delivery Platforms. J. Mater. Chem. в 2013, 14, 401-428.

4 Montellano, A.; Da Ros, T.; Bianco, A.; Prato, M. Fullerene $C_{60}$ as a Multifunctional System for Drug and Gene Delivery. Nanoscale 2011, 3, 40354041.

5 Lyon, D. Y.; Fortner, J. D.; Sayes, C. M.; Colvin, V. L.; Hughes, J. B. Bacterial Cell Association and Antimicrobial Activity of a $\mathrm{C}_{60}$ Water Suspension. Environ. Toxicol. Chem. 2005, 24, 2757-2762.

6 Lyon, D. Y.; Brown, D. A.; Alvarez, P. J. J. Implications and Potential Applications of Bactericidal Fullerene Water Suspensions: Effect on $\mathrm{nC}_{60}$ Concentration, Exposure Conditions and shelf Life. Water Sci. Technol. 2008, $57,1533-1538$.

$7 \mathrm{Ma}$, Y. Q. Theoretical and Computational Studies of Dendrimers as Delivery Vectors. Chem. Soc. Rev. 2013, 42, 705-727. 
8 Ding, H. M.; Ma, Y. Q. Theoretical and Computational Investigations of Nanoparticle-Biomembrane Interactions in Cellular Delivery. Small 2015, 11, 1055-1071.

9 Fortner, J. D.; Lyon, D. Y.; Sayes, C. M.; Boyd A. M.; Falkner, J. C.; Hotze, E. M.; Alemany, L. B.; Tao, Y. J.; Guo, W.; Ausman, et al. $C_{60}$ in Water: Nanocrystal Formation and Microbial Response. Environ. Sci. Technol. Lett. 2005, 39, 43074316.

10 Lyon, D. Y.; Alvarez. P. J. J. Fullerene Water Suspension ( $\mathrm{nC}_{60}$ ) Exerts Antibacterial Effects via ROS-Independent Protein Oxidation. Environ. Sci. Technol. Lett. 2008, 42, 8127-8132.

11 Brunet, L.; Lyon, D. Y.; Hotze, E. M.; Alvarez, P. J.; Wiesner, M. R. Comparative Photoactivity and Antibacterial Properties of $\mathrm{C}_{60}$ Fullerenes and Titanium Dioxide Nanoparticles. Environ. Sci. Technol. 2009, 43, 4355-4360.

12 Berglund, N. A.; Piggot, T. J.; Jefferies, D.; Sessions, R. B.; Bond, P. J.; Khalid, S. Interaction of the Antimicrobial Peptide Polymyxin B1 with Both Membranes of E. coli: a Molecular Dynamics Study. PLoS Comput. Biol. 2015, 11, e1004180.

13 Sansom, M. P.; Scott, K.; Bond, P. Coarse-Grained Simulation: a HighThroughput Computational Approach to Membrane Proteins. Biochem. Soc. Trans. 2008, 36, 27-32.

14 Chen, K.; Ma, Y. Q. Interaction of Fullerene Chains and a Lipid Membrane via Computer Simulations. RSC Adv. 2014, 4, 30215-20.

15 D’Rozario, R. S.; Wee, C. L.; Wallace, E. J.; Sansom, M. S. The Interaction of C60 and its Derivatives with a Lipid Bilayer via Molecular Dynamics Simulations. Nanotechnol. 2009, 20, 115102.

16 Wong-Ekkabut, J.; Baoukina,S.; Triampo, W.; Tang, I. M.; Tieleman, D. P.; Monticelli, L. Computer Simulation Study of Fullerene Translocation Through Lipid Membranes. Nat. Nanotechnol. 2008, 3, 363-368.

17 DeVane, R.; Jusufi, A.; Shinoda, W.; Chiu, C. C.; Nielsen, S. O.; Moore, P. B.; Klein, M. L. Parameterization and Application of a Coarse Grained Force Field for Benzene/Fullerene Interactions with Lipids. J. Phys. Chem. B 2010, 114, $16364-16372$. 
18 Zähringer, U.; Lindner, B.; Seydel, U.; Rietschel, E. T.; Naoki, H.; Unger, F. M.; Imoto, M.; Kusumoto, S.; Shiba, T. Structure of De-O-acylated Lipopolysaccharide from the Escherichia Coli Re Mutant Strain F 515. Tetrahedron Lett. 1985, 26, 6321-6324.

19 Mclntire, F. C.; Sievert, H. W.; Barlow, G. H.; Finley, R. A.; Lee, A. Y. Chemical, Physical, and Biological Properties of a Lipopolysaccharide from Escherichia Coli K-235. Biochemistry 1967, 6, 2363-2372.

20 Ma, H.; Irudayanathan, F. J.; Jiang, W.; Nangia, S. Simulating Gram-Negative Bacterial Outer Membrane: a Coarse Grain Model. J. Phys. Chem. B 2015, 119, 14668-14682.

21 Van Oosten, B.; Harroun, T. A. A MARTINI Extension for Pseudomonas Aeruginosa PAO1 Lipopolysaccharide. J. Mol. Graph. 2016, 63, 125-133.

22 Nikaido, H. Molecular Basis of Bacterial Outer Membrane Permeability Revisited. Microbiol. Mol. Biol. Rev. 2003, 67, 593-656.

23 Nikaido, H.; Vaara, M. Molecular Basis of Bacterial Outer Membrane Permeability. Microbiol. Rev. 1985, 49, 1.

24 Clifton, L. A.; Holt, S. A.; Hughes, A. V.; Daulton, E. L.; Arunmanee, W.; Heinrich, F.; Khalid, S.; Jefferies, D.; Charlton, T. R.; Webster, J. R. et al. An Accurate in Vitro Model of the E. Coli Envelope. Angew. Chem. Int. Ed. 2015, 54, $11952-19955$.

25 Szczelina, R.; Murzyn, K. DMG- $\alpha$ A Computational Geometry Library for Mulitmolecular Systems. J. Chem. Inf. Model. 2014, 54, 3112-3123.

26 Parrinello, M.; Rahman, A. Polymorphic Transitions in Single Crystals: a New Molecular Dynamics Method. J. Appl. Phys. 1981, 52, 7182-7190.

27 Nosé, S.; Klein, M. L. Constant Pressure Molecular Dynamics for Molecular Systems. Mol. Phys. 1983, 50, 1055-1076.

28 Monticelli, L. On Atomistic and Coarse-Grained Models for $\mathrm{C}_{60}$ Fullerene. J. Chem. Theory Comput. 2012, 8, 1370-1378.

29 Humphrey, W.; Dalke, A.; Schulten, K. VMD: Visual Molecular Dynamics. J. Mol. Graph. 1996, 14, 33-38. 
30 Castillo, N.; Monticelli, L.; Barnoud, J.; Tieleman, D. P. Free Energy of WALP23 Dimer Association in DMPC, DPPC, and DOPC Bilayers. Chem. Phys. Lipids 2013, 169, 95-105.

31 Ikeda, A.; Mori, M.; Kiguchi, K.; Yasuhara, K.; Kikuchi, J. I.; Nobusawa, K.; Akiyama, M.; Hashizume, M.; Ogawa, T.; Takeya, T. Advantages and Potential of Lipid-Membrane-Incorporating Fullerenes Prepared by the FullereneExchange Method. Chem. Asian J. 2012, 7, 605.

32 Ikeda, A.; Sato, T.; Kitamura, K.; Nishiguchi, K.; Sasaki, Y.; Kikuchi, J. I.; Ogawa, T.; Yogo, K.; Takeya, T. Efficient Photocleavage of DNA Utilizing Water-Soluble Lipid Membrane-Incorporated [60] Fullerenes Prepared Using a [60] Fullerene Exchange Method. Org. Biomol. Chem. 2005, 16, 2907-2909.

33 Ikeda, A.; Doi, Y.; Nishiguchi K.; Kitamura, K.; Hashizume, M.; Kikuchi, J. I.; Yogo, K.; Ogawa, T. Takeya, T. Induction of Cell Death by Photodynamic Therapy with Water-Soluble Lipid-Membrane-Incorporated [60] Fullerene. Org. Biomol. Chem. 2007, 5, 1158-1160.

34 Monticelli, L. Bozdaganyan, M. E.; Orekhov, P. S.; Shaytan, A. K.; Shaitan, K. V. Comparative Computational Study of Interaction of $\mathrm{C}_{60}$-Fullerene and TrisMalonyl- $\mathrm{C}_{60}$-Fullerene Isomers with Lipid Bilayers: Relation to their Antioxidant Effect. PLOS ONE 2014, 9, e102487.

35 Bedrov, D.; Smith, G. D.; Davande, H.; Li, L. Passive Transport of $C_{60}$ Fullerenes Through a Lipid Membrane: a Molecular Dynamics Simulation Study, J. Phys. Chem. B 2008, 112, 2078-2084.

36 Deguchi, S.; Alargova, R. G.; Tsujii, K. Stable Dispersions of Fullerenes, $C_{60}$ and $\mathrm{C}_{70}$, in Water Preparation and Characterization. Langmuir 2001, 17, 60136017.

37 Chiu, C. C.; DeVane, R.; Klein, M. L.; Shinoda, W.; Moore, P. B.; Nielsen, S. O. Coarse-Grained Potential Models for Phenyl-Based Molecules: II. Application to Fullerenes. J. Phys. Chem. B 2010, 114, 6394-6400.

38 Snyder, S.; Kim, D.; McIntosh, T. J. Lipopolysaccharide Bilayer Structure: Effect on Chemotype, Core Mutations, Divalent Cations, and Temperature. Biochemistry 1999, 38, 10758-10767. 
39 Nascimento, A.; Pontes, F. J.; Lins, R. D.; Soares, T. A. Hydration, Ionic Valence and Cross-Linking Propensities of Cations Determine the Stability of Lipopolysaccharide (LPS) Membranes. Chem. Commun. 2014, 50, 231-233

40 Nikaido, H. Permeability of the Lipid Domains of Bacterial Membranes. Adv. Membr. Fluid 1990, 4, 165-190.

41 Vaara, M.; Nurminen, M. Outer Membrane Permeability Barrier in Escherichia coli Mutants that are Defective in the Late Acyltransferases of Lipid A Biosynthesis, Antimicrob. Agents Chemother. 1999, 43, 1459-1462.

42 Vaara, M. Antibiotic-Supersusceptible Mutants of Escherichia Coli and Salmonella Typhimurium. Antimicrob. Agents Chemother. 1993, 37, 2255.

43 Smit, J. O.; Kamio, Y. O.; Nikaido, H. Outer Membrane of Salmonella Typhimurium: Chemical Analysis and Freeze-Fracture Studies with Lipopolysaccharide Mutants. J. Bacteriol. 1975, 124, 942-958.

44 Kubiak, J.; Brewer, J.; Hansen, S.; Bagatolli, L. A. Lipid Lateral Organization on Giant Unilamellar Vesicles Containing Lipopolysaccharides. Biophys. J. 2011, 100, 978-986.

45 Barnoud, J.; Rossi, G.; Monticelli, L. Lipid Membranes as Solvents for Carbon Nanoparticles, Phys. Rev. Lett. 2014, 112, 068102.

46 Trabelsi, S.; Zhang, S.; Lee, T. R.; Schwartz, D. K. Linactants: Surfactant Analogues in Two Dimensions. Phys. Rev. Lett. 2008, 100, 037802.

47 Schäfer, L. V.; Marrink, S. J. Partitioning of Lipids at Domain Boundaries in Model Membranes. Biophys. J. 2010, 99, L91-L93.

48 Barnoud, J.; Rossi G.; Marrink, S. J.; Monticelli, L. Hydrophobic Compounds Reshape Membrane Domains. PLoS Comput. Biol. 2014, 10, e1003873.

49 Palmieri, B.; Yamamoto, T.; Brewster, R. C.; Safran, S. A. Line Active Molecules Promote Inhomogeneous Structure in Membranes: Theory, Simulations and Experiments. Adv. Colloid Interface Sci. 2014, 208, 58-65.

50 Garcia-Sáez, A. J.; Chiantia, S.; Schwille, P. Effect of Line Tension on the Lateral Organization of Lipid Membranes. J. Biol. Chem. 2007, 282, 3353733544. 
51 Hadduck, A. N.; Hindagolla, V.; Contreras, A. E.; Li, Q.; Bakalinsky, A. T. Does Aqueous Fullerene Inhibit the Growth of Saccharomyces Cerevisiae or Escherichia Coli? Appl. Environ. Microbial. 2010, 76, 8239-8242.

52 Kang, S.; Mauter, M. S.; Elimelech, M. Microbial Cytotoxicity of Carbon-Based Nanomaterials: Implications for River Water and Wastewater Effluent. Environ. Sci. Technol. 2009, 43, 2648-2653.

53 Chiron, J. P.; Lamande, J.; Moussa, F.; Trivin, F.; Céolin, R. Effect of "Micronized" $\mathrm{C}_{60}$ Fullerene on the Microbial Growth in Vitro, Ann. Pharm. Fr. 2000, 58, 170-175.

54 An, H.; Jin, B. Fullerenols and Fullerene Alter Cell Growth and Metabolisms of Escherichia Coli. J. Biomed. Nanotechnol. 2015, 11, 1261-1268. 


\section{TOC Graphic}

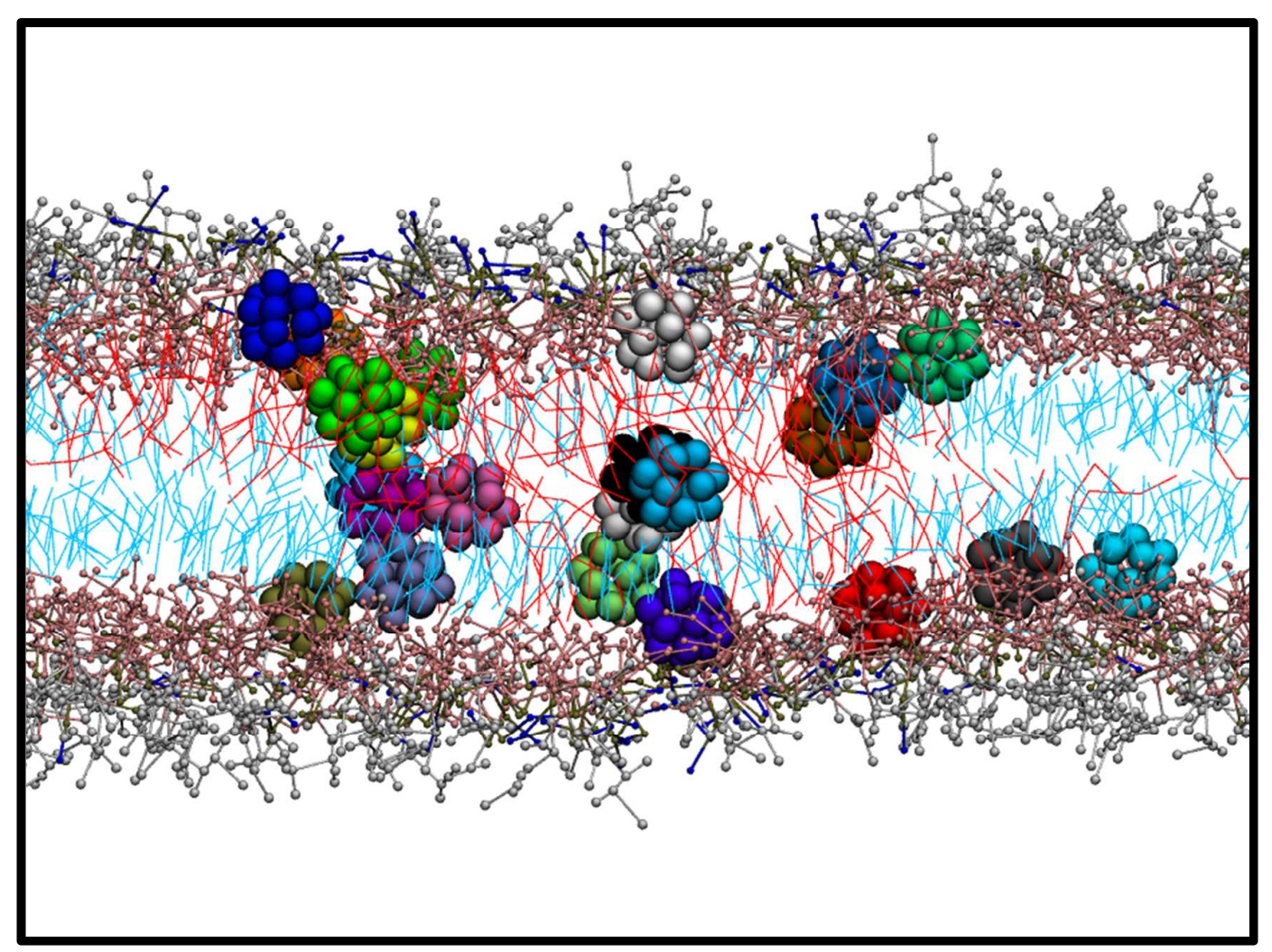



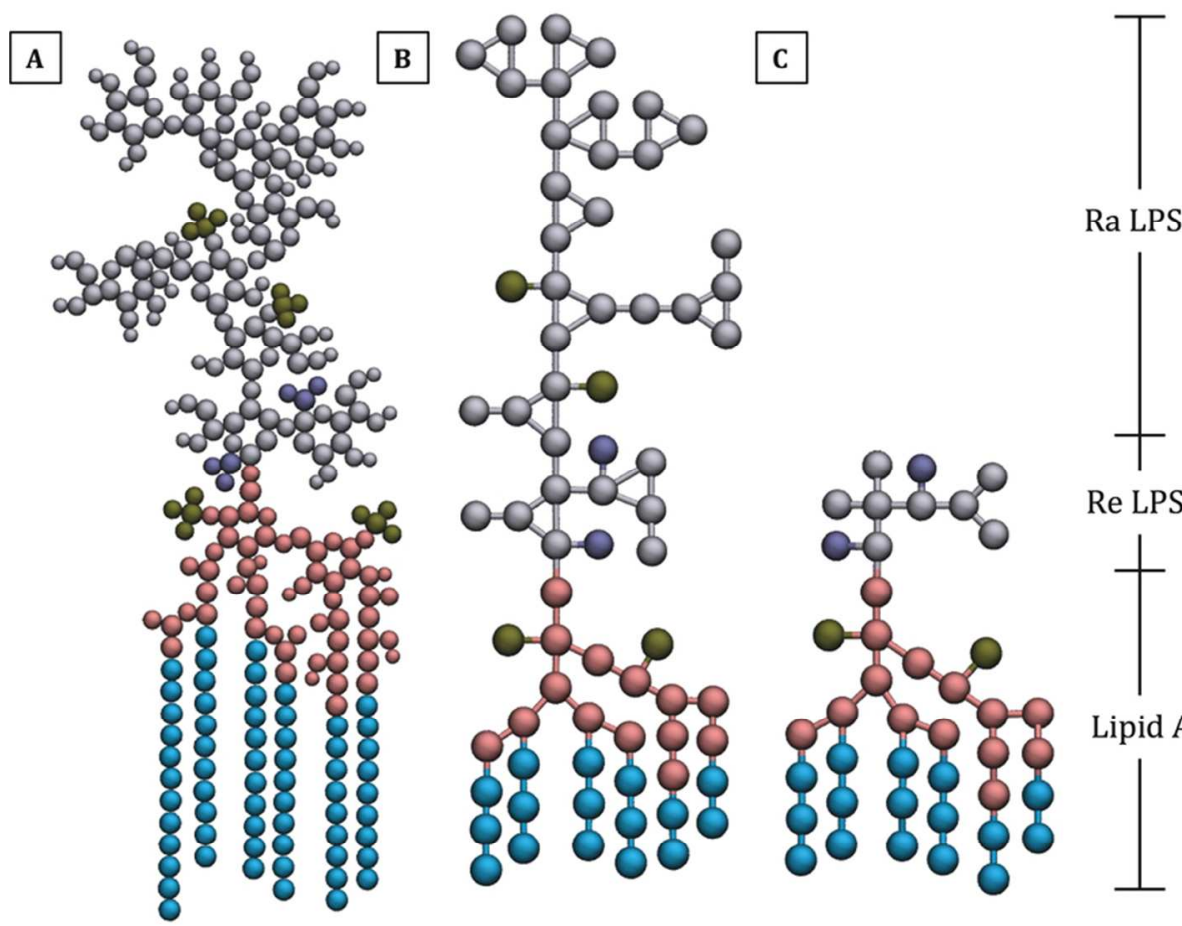

Re LPS

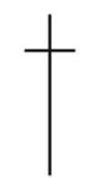

Lipid A

Figure 1: CG mapping schemes for Re and Ra LPS (A) The UA model for Ra LPS is shown alongside the CG model for (B) Ra LPS, and (C) Re LPS. Carbon tails are colored cyan, phosphate groups are colored brown, carboxylate groups are colored dark blue, glucosamine and glycerol groups are colored pink, and the remaining core saccharide sections are colored silver. Figure 1

$81 \times 61 \mathrm{~mm}(300 \times 300 \mathrm{DPI})$ 
Figure 2: Comparative partial mass density plots for E. coli outer membranes. E. coli membranes were simulated at $310 \mathrm{~K}$ using CG and UA representations. The first membrane was built with a leaflet of $(A)$ Re LPS, while the second membrane contained a leaflet of (B) Ra LPS instead. Carbon tails are represented by black lines, glucosamine, glycerol and ethanolamine groups (Glc,Gly,Eth) are represented by green lines, phosphate groups are represented by turquoise lines, calcium ions are represented by pink lines, inner core sugars are represented by brown lines, outer core sugars are represented by orange lines, and water is represented by blue lines. The partial mass densities are measured along the bilayer normal ( $Z$ axis).

Figure 2

$114 \times 152 \mathrm{~mm}(150 \times 150 \mathrm{DPI})$ 
1

2

3

4

5

6

7

8

9

10

11

12

13

14

15

16

17

18

19

20

21

22

23

24

25

26

27

28

29

30

31

32

33

34

35

36

37

38

39

40

41

42

43

44

45

46

47

48

49

50

51

52

53

54

55

56

57

58

59

60
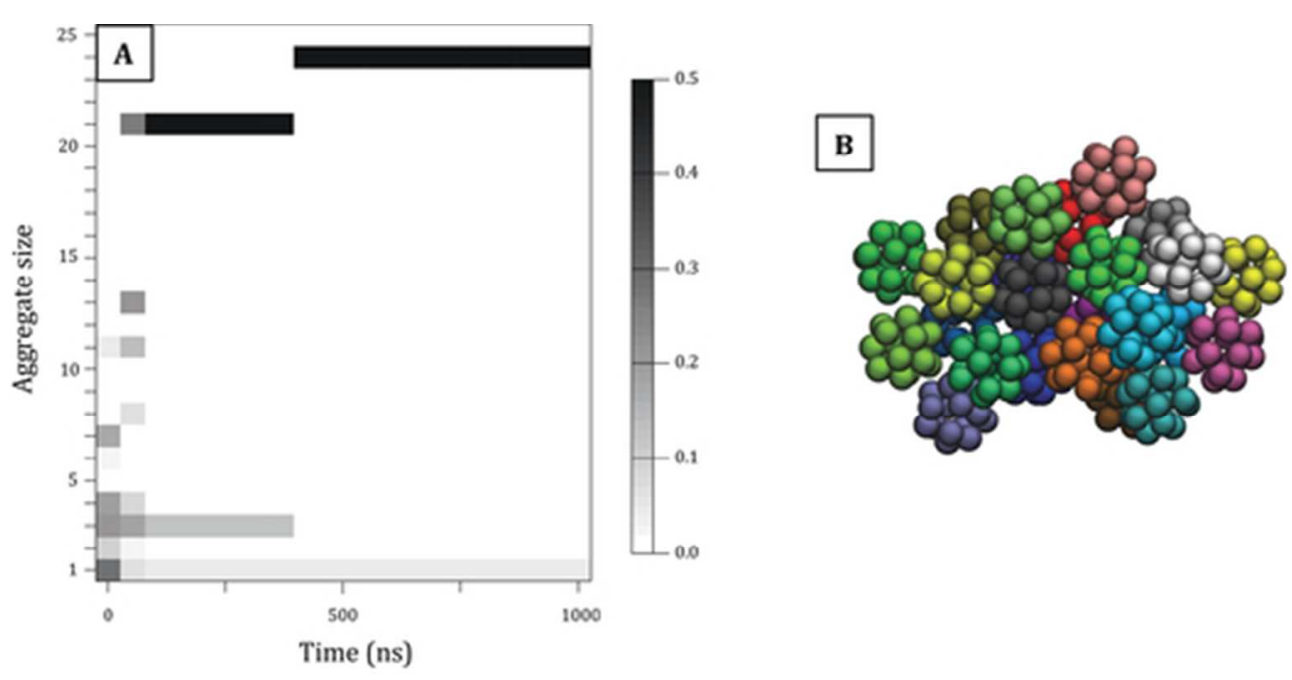

Figure 3: Clustering of $C 60$ in a representative CG simulation (A) The clustering of C60 is plotted as a function of time during the first $1 \mu$ s of a CG simulation. The grayscale in each cell represents the fraction of C60 involved in an aggregate of a given size ( $y$ axis) at a given time (x axis). The C60 concentration was $1.13 \mathrm{mM}$. (B) Snapshot of the 24-unit C60 cluster formed toward the end of this initial period of aggregation.

Figure 3

$45 \times 22 \mathrm{~mm}(300 \times 300 \mathrm{DPI})$ 

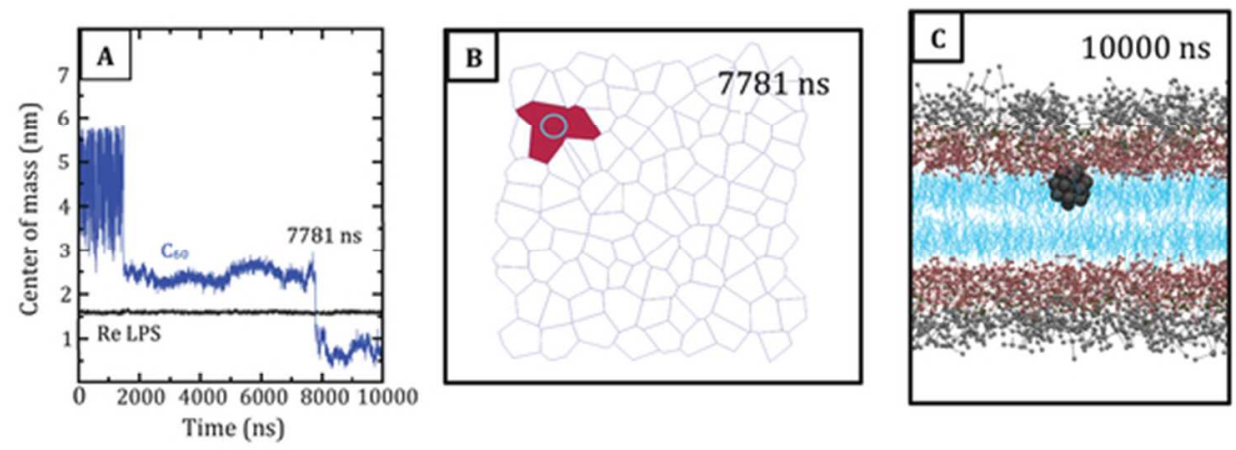

Figure 4: The effect of sodium ions on Re LPS bilayer permeability. (A) Position of the C60 monomer along the bilayer normal, relative to the Re LPS bilayer center. The blue line depicts the center of mass coordinates for the C60 monomer from the bilayer center, while the black line shows the average position of LPS boundary phosphate groups in a single leaflet of the bilayer. The simulation was performed at $310 \mathrm{~K}$, with a single C60 fullerene. (B) Two-dimensional voronoi tessellation of the Re LPS surface as the C60 fullerene bypasses the phosphate barrier at $7781 \mathrm{~ns}$. Each cell represents a single Re LPS head group. The red cells correspond to the LPS head groups adjacent to the C60 monomer. The cyan circle shows the position of the C60 monomer during the head group tunneling event. (C) Side-view snapshot for the C60 monomer after it bypassed the phosphate interface to settle in the lipid core.

Figure 4

$51 \times 19 \mathrm{~mm}(300 \times 300$ DPI $)$ 

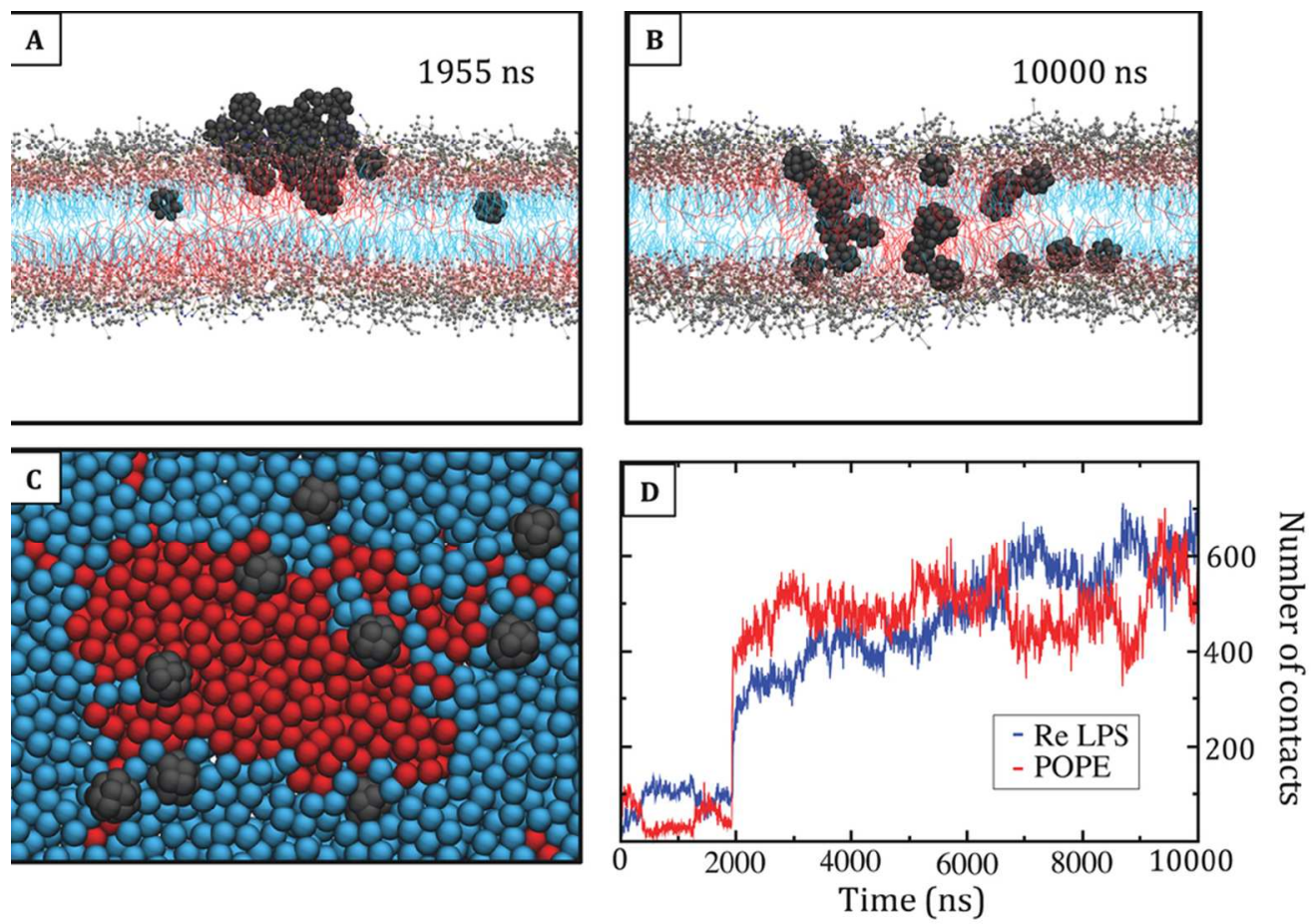

Figure 5: Migration of $\mathrm{C} 60$ fullerenes to the POPE-Re LPS lipid interface. (A) The C60 cluster migrates to the domain of POPE, and slips past the hydrophilic head groups to access the lipid core. (B) The cluster disaggregates, and the disbanded fullerenes drift to the interface between the separate POPE and LPS domains. The Re LPS are colored as before, and the POPE lipid tails are colored red. (C) Top-view snapshot of the disbanded fullerenes at the POPE-Re LPS lipid interface. The lipid head groups are omitted for clarity. (D) The number of contacts between the C60 fullerenes and the (Re LPS and POPE) acyl tails is plotted as a function of simulation time. The red line shows the number of contacts between the fullerenes and POPE lipid tails, while the blue shows the number of contacts between the fullerenes and the Re LPS lipid tails. The relative number of contacts was determined using a $0.6 \mathrm{~nm}$ cutoff distance.

Figure 5

$85 \times 61 \mathrm{~mm}(300 \times 300$ DPI $)$ 

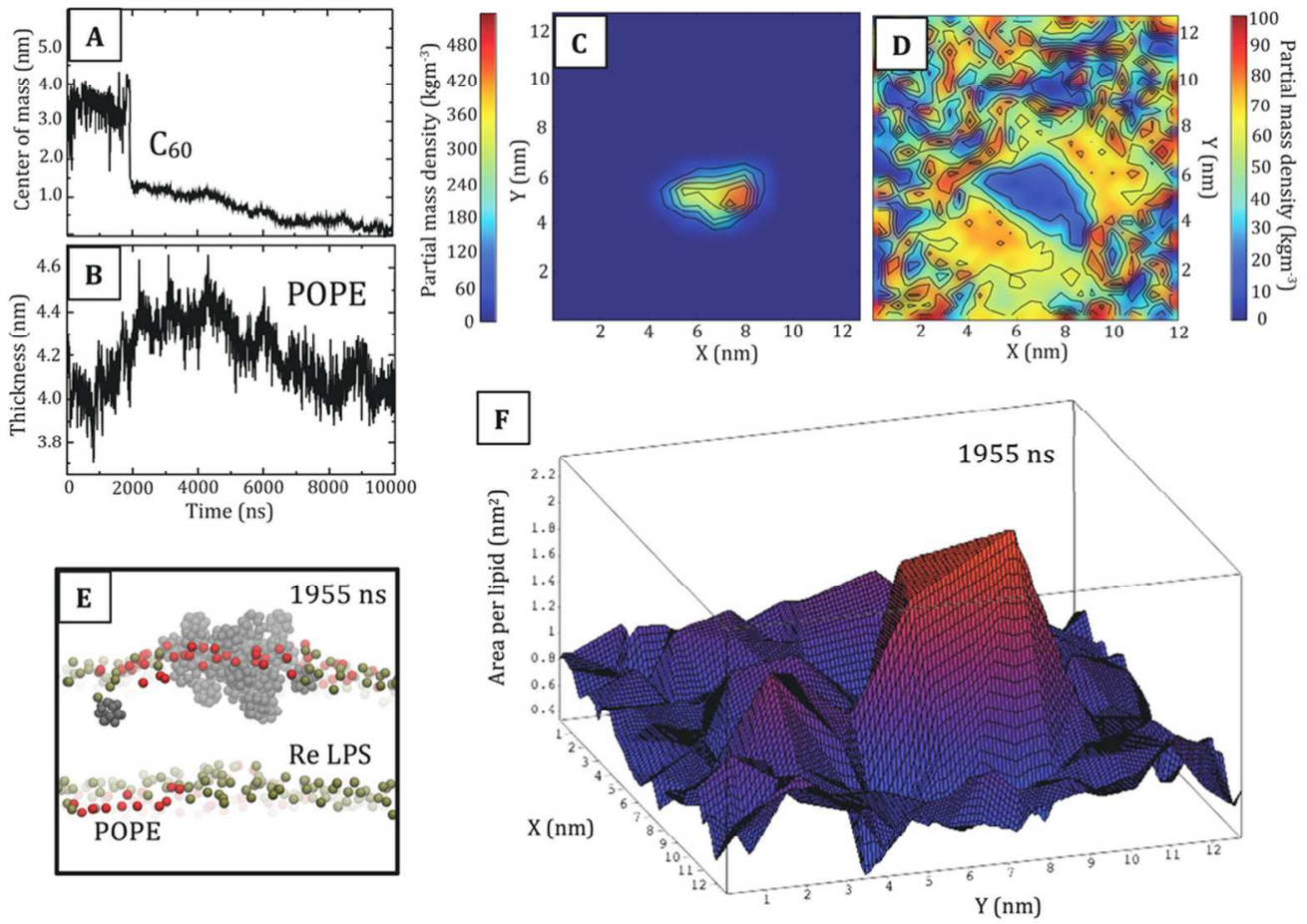

Figure 6: Translocation of a $\mathrm{C} 60$ cluster across the water-lipid interface. (A) Average center of mass coordinates for $\mathrm{C} 60$ fullerenes along the bilayer normal (relative to the bilayer center). (B) Average thickness of the POPE lipids plotted against simulation time; thickness was defined as the distance between POPE phosphate head groups and the bilayer center. (C) Two-dimensional partial mass density for C60 fullerenes comprising the C60 cluster, during the bilayer insertion event (1955 ns). (D) Partial mass densities for Re LPS phosphate and POPE choline head groups during fullerene cluster insertion (1955 ns). The POPE head groups move apart to create a transient micropore whose shape and projected surface area match those of the C60 cluster. (E) Side-view snapshot of the C60 cluster traversing the phosphate interface, the Re LPS phosphate groups are colored brown, the POPE phosphate groups are colored red. All other membrane components are omitted for clarity. (F) Three-dimensional projection of area per lipid values for the membrane leaflet that the $\mathrm{C} 60$ fullerenes pass through. The tessellation was performed for the membrane surface at 1955 ns. Red color represents high area per lipid while blue color represents low area per lipid.

Figure 6

$92 \times 70 \mathrm{~mm}(300 \times 300 \mathrm{DPI})$ 

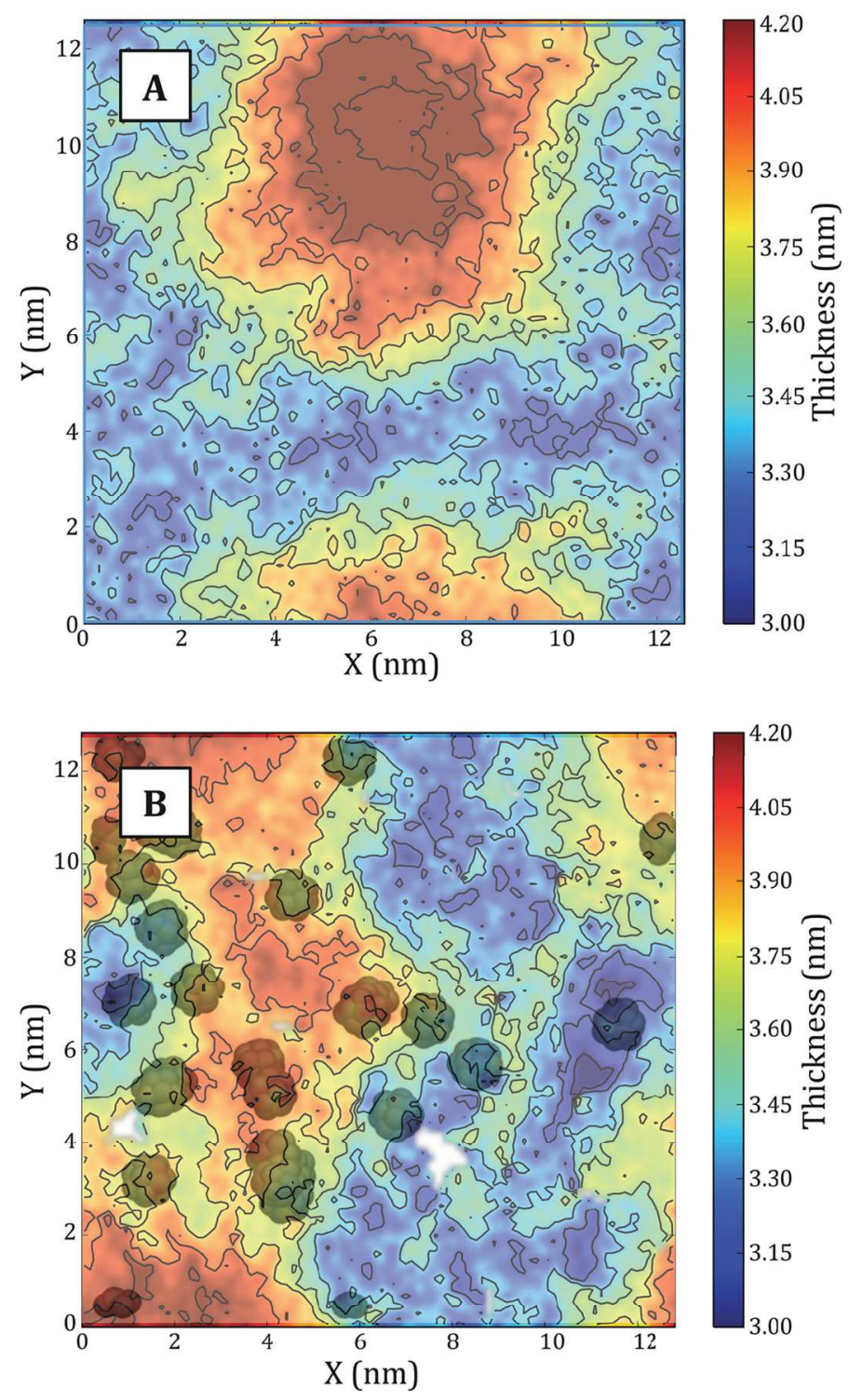

C60 fullerenes promote lipid mixing in the multicomponent membrane. After the C60 fullerenes had entered the bilayer core, they migrated to the boundaries between the coexisting POPE and Re LPS domains. The fullerenes then reduced the line tension between the coexisting lipid fractions by minimizing structural mismatch. The figures depict the bilayer thickness as a two-dimensional landscape for the membrane (A) without any C60 fullerenes dissolved in the lipid core, and (B) with 25 C60 fullerenes dispersed among the lipid tails. The final $\mathrm{C} 60$ particle positions are depicted above the thickness landscape as translucent black spheres (for reference).

Figure 7

$105 \times 170 \mathrm{~mm}(300 \times 300 \mathrm{DPI})$ 


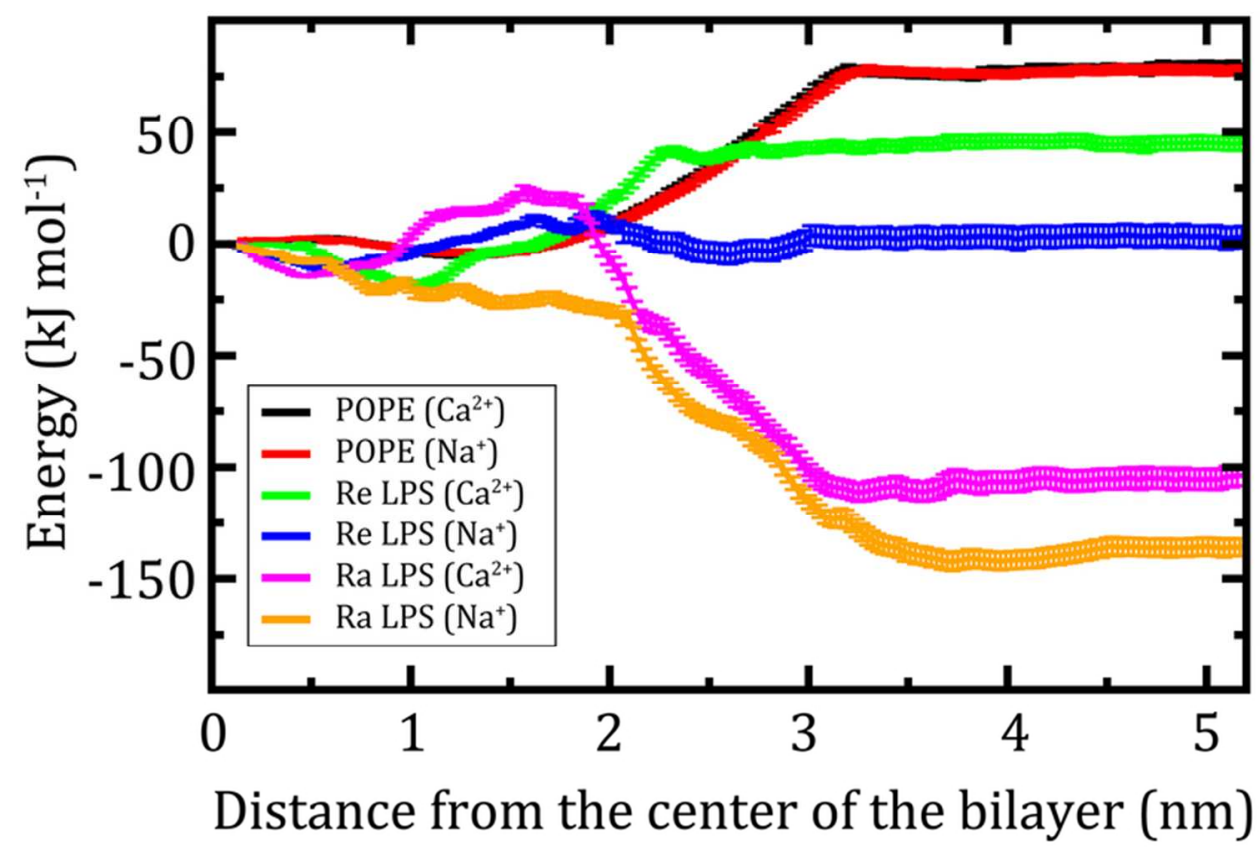

Figure 8: PMF profiles for C60 fullerene as a function of distance from the center of homogeneous lipid bilayers. PMF profiles for $\mathrm{C} 60$ fullerene as a function of distance from the bilayer center. The red line shows the data for the POPE bilayer with $\mathrm{Na}+$ ions, the black line shows the data for the POPE bilayer with Ca2+, the green line shows the data for the Re LPS bilayer with Ca2+ ions, the blue line shows the data for the Re LPS bilayer with $\mathrm{Na}+$, the pink line shows the data for the Ra LPS bilayer with $\mathrm{Ca} 2+$ ions, and the orange line shows the data for the Ra LPS bilayer with $\mathrm{Na}+$ ions. Each umbrella sampling simulation was $1 \mu$ long; the simulation temperature was $310 \mathrm{~K}$; the umbrella sampling windows were separated by distances of 0.2 $\mathrm{nm}$. The free energy was set to zero at the bilayer center.

Fig 8

$37 \times 29 \mathrm{~mm}(600 \times 600 \mathrm{DPI})$ 